\title{
Modelo de gestión del riesgo en proyectos informáticos Mogripi
}

Model of risk management in IT projects Mogripi 


\title{
Modelo de gestión del riesgo en proyectos informáticos Mogripi ${ }^{1}$ Model of risk management in IT projects Mogripi
}

\author{
Ricardo Jesús Barrera², Maritza del Pilar Sánchez Delgado³, \\ William Mauricio Rojas Contreras ${ }^{4}$ \\ Universidad de Pamplona, Pamplona, Colombia
}

Artículo recibido en marzo de 2016; artículo aceptado en junio de 2016

Citación del artículo: Barrera, R.; Sánchez, M. \& Rojas, W. (2016). Modelo de gestión del

riesgo en proyectos informáticos Mogripi. I+D Revista de Investigaciones, 8(2), pp. 15 - 24

\section{Resumen}

El presente artículo explica el modelo de gestión del riesgo en proyectos informáticos Mogripi creado a partir de dos enfoques principales: Gestión de Proyectos (PMBOK ${ }^{\circledR}, 2013$ ) y Gestión del Riesgo (PMBOK ${ }^{\circledR}, 2013$; NTC ISO $31000{ }^{\circledR}$, 2011; COBIT ${ }^{\circledR}$, 2012). En el artículo se utilizará el siguiente esquema: resumen, introducción, metodología, resultados, discusión y referencias. En cuanto a la investigación, en la introducción se explica su propósito e importancia, en la metodología se explica cómo se llevó a cabo, en los resultados se exponen los datos obtenidos y finalmente, en la discusión, se explican los resultados y se compara con el conocimiento previo.

La falta de gestión de los riesgos en proyectos informáticos es un agente causante de fracaso en la terminación exitosa de estos. En este sentido, los resultados de validación del modelo Mogripi permitieron establecer que a través de las tres actividades principales: preparar, valorar y tratar los riesgos, se aumenta la posibilidad de alcanzar el cumplimiento de los objetivos del proyecto. La aplicación de los resultados de la investigación se llevó a cabo en la organización Caja de Compensación Familiar de Norte de Santander, Comfanorte.

Palabras clave: gestión del riesgo, gestión de proyectos.

\footnotetext{
Abstract

The present article explains the model of risk management in IT projects Mogripi created from two main approaches: Project Management (PMBOK $\left.{ }^{\circledR}, 2013\right)$ and Risk Management (PMBOK ${ }^{\circledR}, 2013$; NTC ISO 31000 ${ }^{\circledR}, 2011$, COBIT $^{\circledR}$, 2012). In the article the following scheme is used: Abstract, Introduction, Methods, Results, Discussion and bibliography. A level

1. El artículo tiene un enfoque cualitativo, y es el resultado de un proyecto de investigación en curso perteneciente al área de Gestión de Proyectos, subárea de Gestión de Riesgos, desarrollado en el grupo de investigación Ciencias Computacionales; fue financiado por la Universidad Pamplona de la ciudad de Pamplona, Colombia. Dirección: km. 1 vía Bucaramanga, PBX: 5685303. Fecha de inicio: abril de 2014, fecha de terminación: abril de 2016.

2. Ingeniero de Sistemas, Universidad Francisco de Paula Santander, Cúcuta Colombia. Magister (C) en Gestión de Proyectos Informáticos, Universidad de Pamplona de la ciudad de Pamplona, Colombia. Dirección: km. 1 vía Bucaramanga, PBX: 5685303. Correo electrónico: rj.barrera@ outlook.com

3. Ingeniero de Sistemas, Universidad Francisco de Paula Santander, Cúcuta Colombia. Magister en Gestión de Proyectos Informáticos, Universidad de Pamplona de la ciudad de Pamplona, Colombia. Dirección: km. 1 vía Bucaramanga, PBX: 5685303.: pilas@unipamplona.edu.co 4. Ingeniero de Sistemas, Universidad Francisco de Paula Santander, Cúcuta, Colombia. Magister en Ciencias Computacionales, Universidad Autónoma de Bucaramanga, Colombia. Dirección: km. 1 vía Bucaramanga, PBX: 5685303.mrojas@unipamplona.edu.co
} 
of research in introducing the purpose and importance explained, the methodology explained how they conducted, the results obtained data will be presented and finally, in discussing the results are explained and compared with prior knowledge.

The lack of risk management in IT projects constitutes a causative agent failure in the successful completion of the same. The results of model validation Mogripi, They allowed to establish that through the three main activities: Prepare, assess and treat risks; the possibility of achieving compliance with the objectives of the project is increased. The application of the results of research carried out in the organization Family Compensation Fund of Norte de Santander Comfanorte.

Keywords: Risk Management, Project Management.

No es muy común encontrar proyectos terminados a tiempo, dentro del presupuesto planeado y con la calidad esperada; por lo general se cumplen uno o dos de estos requisitos, pero conlleva mucho esfuerzo (Chamoun, 2002). De igual manera, a nivel mundial cada día es mayor el número de organizaciones que trabajan por proyectos y en gran medida el crecimiento futuro de estas organizaciones dependerá de los resultados favorables de estos, y de la generación de nuevos productos o servicios que innoven el mercado (Graham \& England, 1999).

En los proyectos informáticos la gestión de riesgos resulta fundamental para obtener éxito en su desarrollo. Las presiones de la competencia, los cambios normativos y la evolución de las técnicas pueden obligar a los gerentes a modificar lo planeado durante la ejecución de un proyecto. Cambios en los requerimientos de los usuarios, las nuevas herramientas y tecnologías, las constantes amenazas de seguridad y la rotación en el recurso humano, agregan más presión al equipo de trabajo y deterioran la toma de decisiones (Sommerville, 2006).

El propósito de aplicar los conceptos fundamentales de la gestión de proyectos y la gestión de riesgos al modelo propuesto es crear un proceso proactivo de administración de riesgos que abarque el ciclo de vida del proyecto.

El Software Engineering Institute (SEI, 2006) define riesgo como "la posibilidad de sufrir una pérdida" y a la gestión de riesgos como "la práctica compuesta de procesos, métodos y herramientas que posibilita la gestión de los riesgos en un proyecto y que provee de un entorno disciplinado para la toma de decisiones proactiva con base en determinar constantemente que puede ir mal, e identificar cuáles son los riesgos más importantes, enfocarse e implementar estrategias para gestionarlos"; esta actividad se inicia en la primera etapa de un proyecto informático (durante la exploración de conceptos) y se desarrolla durante todo su ciclo de vida (hasta la aceptación del producto del proyecto) (Shari, 2008).Si bien existen varios modelos de gestión del riesgo, este trabajo se basó en la guía de los fundamentos para la dirección de proyectos (Guía del PMBOK , 2013), que plantea seis actividades a realizar en cuanto a la gestión del riesgo: planificar la gestión de riesgos, identificar los riesgos, realizar el análisis cualitativo de los riesgos, realizar el análisis cuantitativo de los riesgos, planificar la respuesta a los riesgos y monitorear y controlar los riesgos (Figura 1).

\section{Descripción General de la Gestión de los Riesgos del Proyecto}

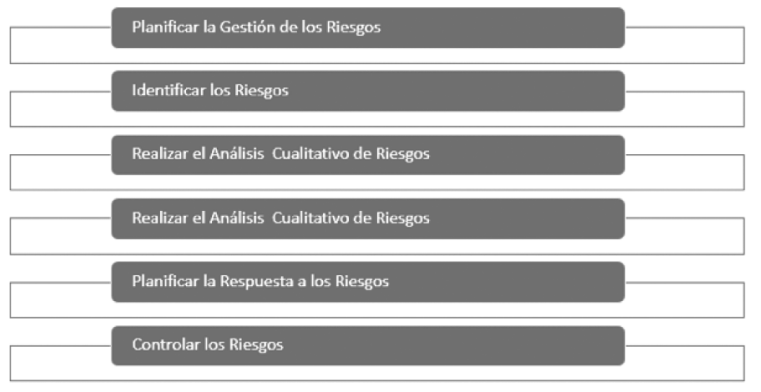

Figura 1.

Procesos para la Gestión del Riesgo (Fuente PMBOK ${ }^{\circledR}$ )

También se tuvo como base la gestión del riesgo planteada por la NTC ISO 31000 (Norma Técnica Colombiana, Institute Standard Organization) en donde se especifican las siguientes etapas: comunicación y consulta, establecimiento del contexto, valoración del riesgo, tratamiento del riesgo, monitoreo y revisión (Instituto Colombiano de Normas Técnicas y Certificación [ICONTEC], 2011) (Figura 2).

La importancia de la presente investigación radica en la facilidad de aplicación del modelo desarrollado, el cual consta de tres procesos fundamentales: preparar la gestión del riesgo, valorar los riesgos y tratamiento 


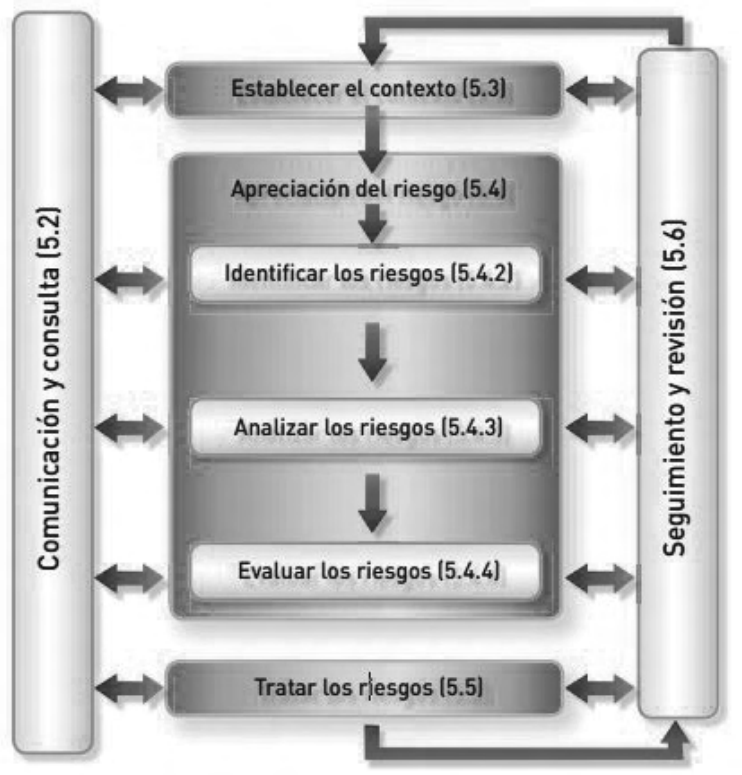

Figura 2.

Proceso para la Gestión del Riesgo. Fuente NTCISO 31000

de los riesgos. Por cada uno de los procesos se tiene un objetivo definido y las actividades claves a realizar para dar cumplimiento con el objetivo trazado.

\section{Método}

Para la presente investigación se planteó un objetivo general: “Diseñar un modelo de gestión de proyectos basándose en las mejores prácticas de estándares existentes orientado a la gestión del riesgo en proyectos informáticos". Este, a su vez, se soporta en tres objetivos específicos:

- $\quad$ Elaboración del estado del arte. Se llevó a cabo una revisión bibliográfica y una revisión electrónica en bases de datos e internet, posteriormente se realizó la integración de referencias.

- Diseño del modelo. Se adelantó una exploración y análisis de técnicas o metodologías para construir modelos, y finalmente se elaboró el modelo Mogripi.

- Validación del modelo. Se utilizó el método analítico y el método de consenso o juicio de expertos Delphi.

La metodología de validación utilizada permite establecer, de acuerdo a la objetividad de la investigación, si el modelo planteado podría ser aplicado en investigaciones con características similares.

En la siguiente figura se ilustra la estructura metodológica empleada para el desarrollo de la investigación.

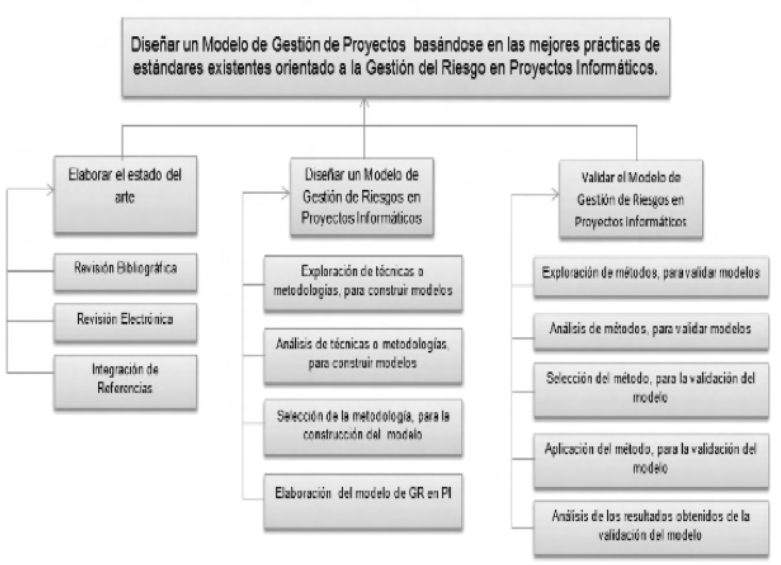

Figura 3.

Estructura metodológica

\section{Evolución de la gestión del riesgo en proyectos}

En esta breve historia de la gestión del riesgo en proyectos, a través de una gráfica, se describen los más grandes desarrollos y eventos, llevándolos tan lejos como hay registros. Se desglosa la gestión del riesgo en generaciones (Marcelo \& Rodenes, 2003) para exponer mejor las características y la evolución de cada una.

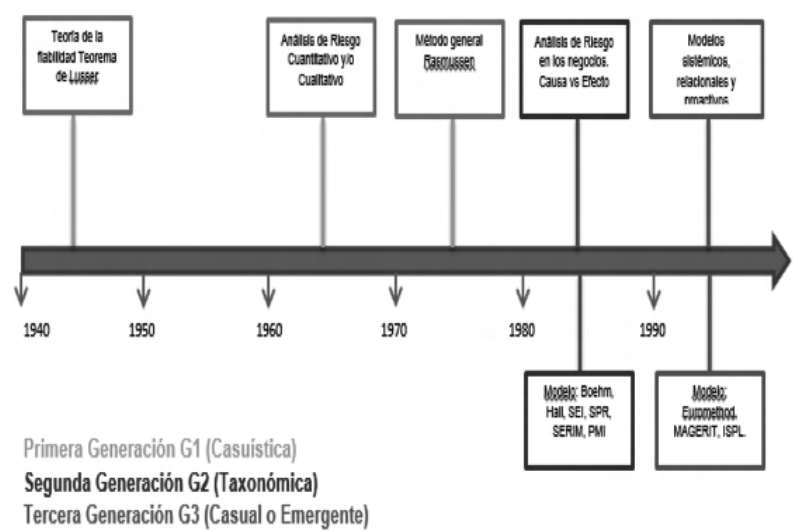

Figura 4.

Evolución histórica de la gestión del riesgo en proyectos

\section{Primera generación G1 (casuística)}

Es la generación casuística o tradicional, donde se limitaban las tareas a la identificación de riesgos en los proyectos con técnicas basadas en cuestionarios, listas de incidencias y de las medidas para contrarrestarlas. Se identificaban casos de riesgo y se extrapolaban a otros proyectos. No hay una planificación específica. En esta generación se definen los riesgos tecnológicos y las listas de comprobación de riesgos. 
Años 40: con la Teoría de la Fiabilidad, arranca la Teoría del Riesgo en Sistemas Complejos, con el Teorema de Lusser: "la probabilidad de éxito (no fallo) de una cadena de componentes es el producto de las probabilidades de éxito de sus elementos".

Años 60: Análisis de riesgos cuantitativo (procesos markovianos) para describir el comportamiento de sistemas complejos con fallos y sin intervención manual (aleatoria); o cualitativos, como los árboles de fallos para sistemas híbridos con la incertidumbre de la intervención humana y la imposibilidad de probar los impactos, salvo por simulación.

Años 70: Método General de Rasmussen con seis etapas: definición del proyecto de seguridad y su sistema objetivo, análisis funcional de este, identificación de riesgos, modelización del sistema, evaluación de consecuencias, síntesis y decisiones finales.

\section{Segunda generación G2 (taxonómica)}

Es la generación taxonómica de análisis de riesgos en los proyectos; traduce a ese sector el análisis de riesgos en los sistemas desarrollado en los ochenta, junto a intentos poco articulados de análisis de riesgo en los negocios.

Los modelos de la G2, que se han limitado a analizar los riesgos al inicio del proyecto y a planificar medidas, definen esta visión como "preventiva", "teorizante" y de medidas "curativas", improvisadas en mayor o menor medida durante el avance del proyecto, para atacar los riesgos según se presenten. Posteriormente, califican a los modelos de la G2 como "meramente reactivos, con unas relaciones de causa-efecto basadas solo en una confianza que parte de experiencias poco validadas". Los marcos contenidos en esta generación son:

- Modelo de Boehm (Barry Boehm en el año 1978).

- $\quad$ Modelo de Hall (Edward T. Hall).

- $\quad$ Modelo del SEl (Software Engineering Institute).

- Modelo SPR de mejora de capacidad en la GR.

- Modelo SERIM (Software Engineering Risk Management) de Karolak: IEEE.

Modelo del PMI (Project Management Institute).

Modelo de McFarlan (Warren McFarland, adelantos de G3 )

\section{Tercera Generación G3 (causal o emergente)}

Es la generación causal o emergente, nacida a mediados de los 90 y referida en particular a proyectos informáticos. Surge de forma simultánea en Europa y en EE. UU., partiendo de la preocupación por proyectos de alto riesgo como la adquisición o el desarrollo de software, y aprovecha los métodos de gestión del riesgo usados en los sistemas. Articula también una causalidad más explicativa y por lo tanto más predictiva entre los elementos del modelo, sobre todo entre los factores de riesgo y sus medidas reductoras o salvaguardas. Esta cuasicausidad, que prepara el paso a la gestión de proyectos por los riesgos, se apoya en modelos sistémicos, relacionales (redes de causas-efectos) y proactivos en el aseguramiento de los proyectos. Los marcos incluidos en esta generación (Cocho \& Adam, 2003):

\section{- $\quad$ Euromethod \\ - Magerit: metodología de análisis y gestión del riesgo de los sistemas de información, Consejo Superior de la Administración Electrónica, España (MAP, 2006). \\ - Information Services Procurement Library (ISPL). \\ - Proyectos de investigación europeos RiskMan, DriveSPI, RiskDriver, Moynihan, Barki, Schmidt e Insead. \\ Modelo para la gestión del riesgo en proyectos informáticos - Mogripi}

Para el desarrollo del modelo se han consultado varias fuentes de información en gestión de proyectos (PMBOK ${ }^{\circledR}$, 2013) y en gestión de los riesgos (PMBOK ${ }^{\circledR}, 2013$; NTCISO 31000, 2011; COBIT 5.0, 2012) (Figura 5).

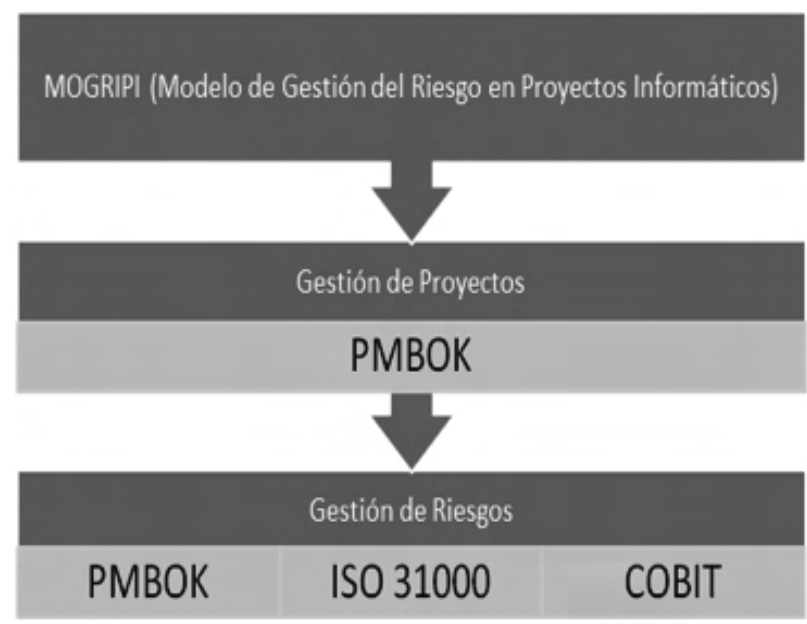

Figura 5.

Fuentes de información de Mogripi 


\section{Estructura del modelo para la gestión del riesgo en proyectos informáticos Mogripi}

El modelo consta de tres procesos fundamentales: preparación de la gestión de los riesgos, valoración de los riesgos y tratamiento de los riesgos. Por cada uno de los procesos se tienen definidos un objetivo y las actividades clave a realizar para dar cumplimiento al objetivo trazado.

Cada actividad tiene entradas y salidas. Se deben establecer medidas para el cumplimiento del objetivo del proceso y para los objetivos de las actividades.

Porcuanto los tres procesos del modelo están supeditados al cumplimiento de los objetivos del proyecto, durante el desarrollo del proyecto es fundamental la comunicación $v$ documentación de los riesaos (Fiaura 6).

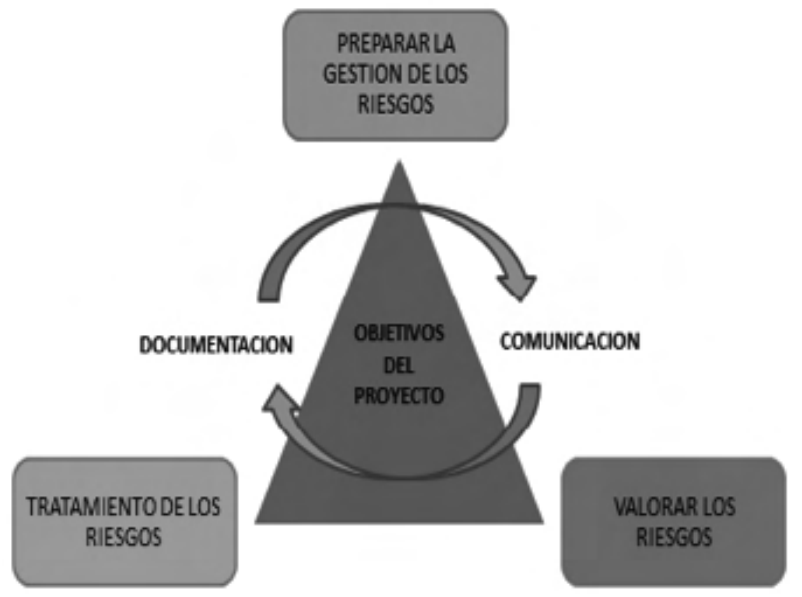

Figura 6.

Procesos de Mogripi

Los objetivos de la gestión de los riesgos del proyecto consisten en aumentar la probabilidad y el impacto de los eventos positivos y disminuir la probabilidad y el impacto de los eventos negativos (PMBOK ${ }^{\oplus}, 2013$ ) (Figura 7).

Para el uso adecuado del modelo Mogripi se debe utilizar la interacción de los tres procesos a través de un marco de referencia para la gestión del riesgo en proyectos informáticos, utilizando como base el ciclo de Deming (PHVA - planear, hacer, verificar, actuar) (Figura 8).

El marco de referencia para la gestión del riesgo en proyectos informáticos permite a la organización adoptar las decisiones de riesgo apropiadas. Dicho marco debe explicar los riesgos y permitir a los usuarios:

Preparar la gestión de los riesgos, permitiendo

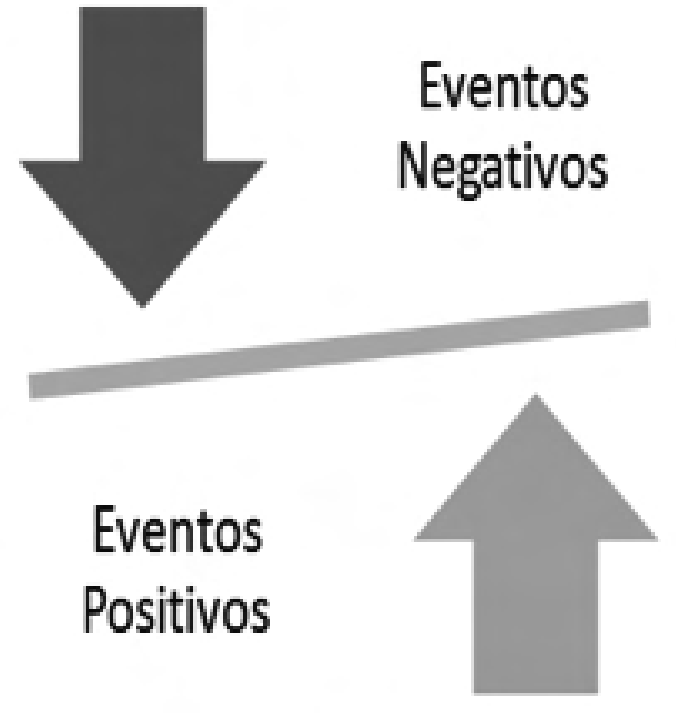

Figura 7.

Objetivos de la gestión del riesgo de Mogripi

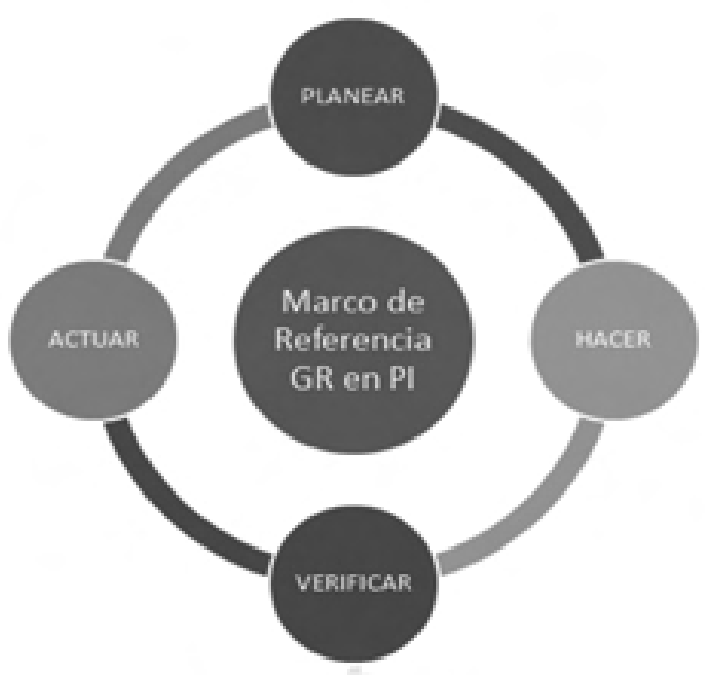

Figura 8.

Marco de Referencia de la Gestión del Riesgo de Mogripi

tomar decisiones conscientes sobre el entorno de los riesgos.

- Valorar los riesgos, adoptando la calificación cuantitativa o cualitativa de los riesgos.

- Tratamiento de los riesgos, entendiendo como responder a los riesgos.

Para un mejor resultado en la gestión del riesgo en proyectos informáticos aplicando Mogripi, durante todo el ciclo de vida del proyecto se debería cumplir con los siguientes principios: 
- $\quad$ Alinearse con los objetivos del proyecto.

- Alinear la gestión de los riesgos de los proyectos informáticos con la gestión de los riesgos de la organización.

- $\quad$ Promover la comunicación y la documentación de la gestión de los riesgos.

- $\quad$ La gestión del riesgo debe promover la mejora continua (Figura 9).

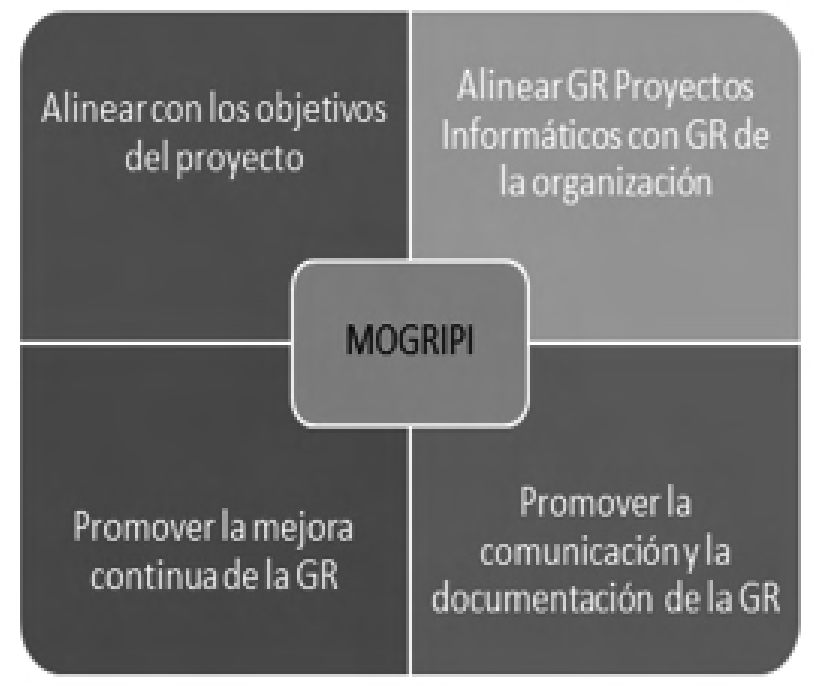

Figura 9.

Principios de la gestión del riesgo de Mogripi

Cada uno de los tres procesos fundamentales del modelo consta de un objetivo, de unas actividades, y de entradas y salidas.

\section{Proceso "Preparar la gestión de los riesgos"}

Objetivo. Realizar actividades concernientes a la preparación de la gestión de los riesgos. Una tarea clave del modelo para este proceso es establecer el contexto externo e interno como una actividad al inicio de este. Al establecer el contexto se definen, entre otros: los objetivos del proyecto, el entorno en el cual se conseguirán los objetivos, las partes involucradas y la diversidad de criterios de riesgo; todo en conjunto ayudará a revelar y evaluar la naturaleza y la complejidad de sus riesgos.

\section{Actividades}

Determinar el contexto externo. Es el ambiente externo, todo aquello fuera del proyecto que pueda influenciar sus objetivos. Tenerlos en consideración es muy importante para elaborar los criterios del riesgo. Algunos de los aspectos a tener en cuenta son: legales, normativos, tecnológicos, financieros, tendencias actuales y partes involucradas.

Determinar el contexto interno. Se refiere al ambiente interno, y está constituido por todo aquello dentro del proyecto que pueda influenciar sus objetivos. Algunos aspectos a tener en cuenta son: políticas, estrategias, recursos humanos, cultura organizacional, procesos, sistemas de información y partes involucradas internas.

Establecer la estrategia de gestión de los riesgos. Se refiere a la definición de la estrategia a utilizar para evaluar la importancia del riesgo. Algunos de los aspectos a tener en cuenta son: naturaleza, causas, consecuencias, probabilidad, nivel de riesgo, apetito de riesgo y tolerancia al riesgo.

\section{Entradas y salidas}

Entradas. Planes de gestión de riesgos en proyectos anteriores y plan del proyecto actual.

Salidas. Estrategia para gestionar los riesgos.

A continuación se ilustra, a través de un diagrama de flujo de datos, el proceso "Preparar la gestión de los riesgos" (Figura 10).

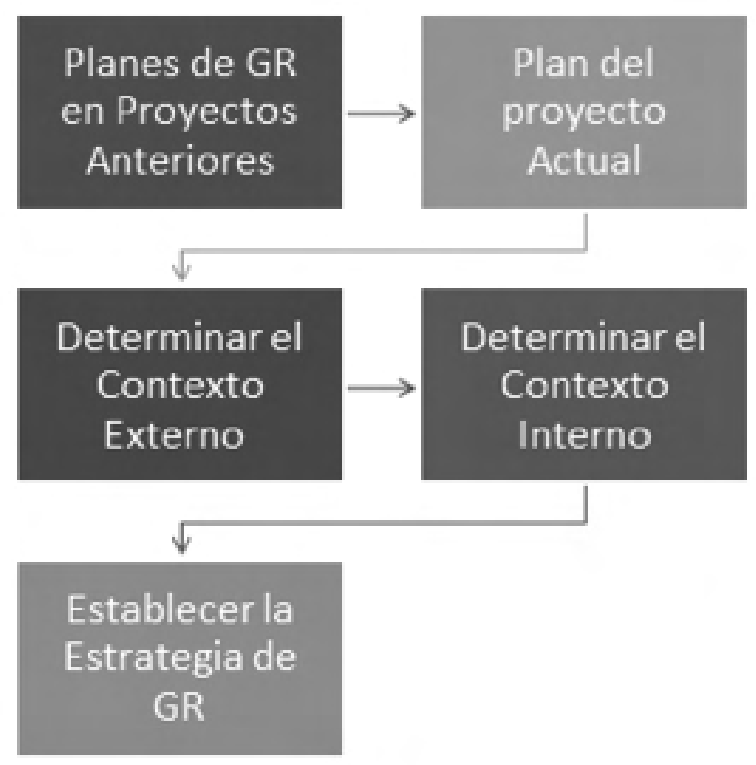

Figura 10.

Diagrama de flujo del proceso: "Preparar la gestión de los riesgos" 


\section{Proceso "Valorar los riesgos"}

Objetivo. Realizar actividades concernientes a la identificación, análisis y evaluación de los riesgos. Una característica clave del modelo para este proceso es la identificación de todo tipo de riesgo, cualquiera sea su naturaleza, bien sea que tenga consecuencias positivas o negativas.

\section{Actividades}

Identificación de los riesgos. Se deben identificar la mayoría de riesgos posibles, elaborando una lista que permita determinar todos los aspectos que puedan impactar positiva o negativamente en el logro de los objetivos del proyecto. Los riesgos se deben clasificar en categorías (estructura de desglose de riesgos). Los aspectos básicos a tener en cuenta son: origen de los riesgos, impacto, causas y consecuencias.

Análisis de los riesgos. El análisis puede ser cualitativo o cuantitativo, dependiendo del contexto del riesgo del proyecto. Se deben tener en cuenta los aspectos que afectan a las consecuencias y la probabilidad.

Evaluación de los riesgos. Una vez realizado el análisis de los riesgos estos deben ser valorados para determinar la prioridad al momento del tratamiento del riesgo.

\section{Entradas y salidas}

Entradas. Estrategia para gestionar los riesgos, categorías de riesgos (estructura de desglose de riegos).

Salidas. Listado de riesgos identificados y valorados, documento técnico (identificación, análisis y valoración de los riesgos).

A continuación se ilustra, a través de un diagrama de flujo de datos, el proceso "Valorar los riesgos" (Figura 11).

\section{Proceso "Tratamiento de los riesgos"}

Objetivo. Realizar actividades concernientes a la respuesta a la gestión de los riesgos. Una característica clave del modelo para este proceso es el establecimiento de controles que modifiquen el riesgo.

\section{Actividades}

Elegir la mejor opción para el tratamiento del riesgo. Se debe elegir la mejor opción o la más adecuada para el tratamiento del riesgo, se debe establecer una relación costo/beneficio de la opción a implementar.

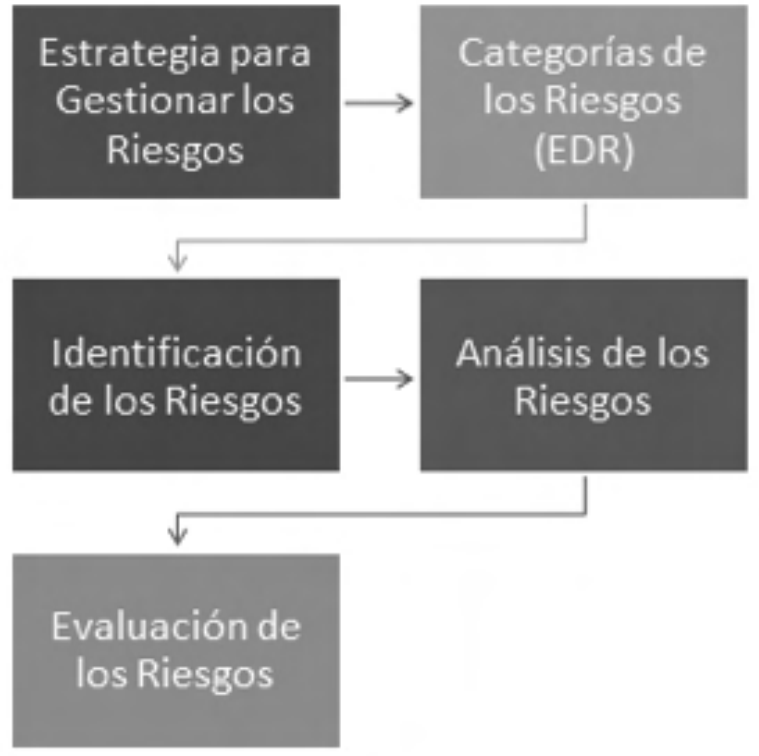

Figura 11.

Diagrama de flujo del proceso: "Valorar los riesgos"

Implementación del tratamiento del riesgo. Se debe generar un documento con las acciones a realizar en el tratamiento de los riesgos. Los aspectos a tener en cuenta son: responsables, recursos, acciones propuestas y tiempo.

\section{Entradas y salidas}

Entradas. Listado de riesgos identificados y valorados, estrategia para el tratamiento de los riesgos.

Salidas. Plan de tratamiento de los riesgos, documento técnico para el seguimiento de los riesgos. Informe de resultados de la gestión de los riesgos.

A continuación se ilustra, a través de un diagrama de flujo de datos, el proceso "Tratamiento de los riesgos" (Figura 12).

\section{Validación del modelo para la gestión del riesgo en proyectos informáticos Mogripi}

A través de la validación del modelo Mogripi se pretende determinar la validez o no de este. El uso de los factores e indicadores preestablecidos definen los criterios de calidad que debe contener el modelo a ser evaluado. Se utilizó el método analítico en la validación del modelo, que consiste en explorar el modelo de forma específica para poder conocer el comportamiento frente a la evaluación de los factores más críticos. De manera consecuente se utilizó el método Delphi, el cual se aplicará a través del juicio de expertos. 


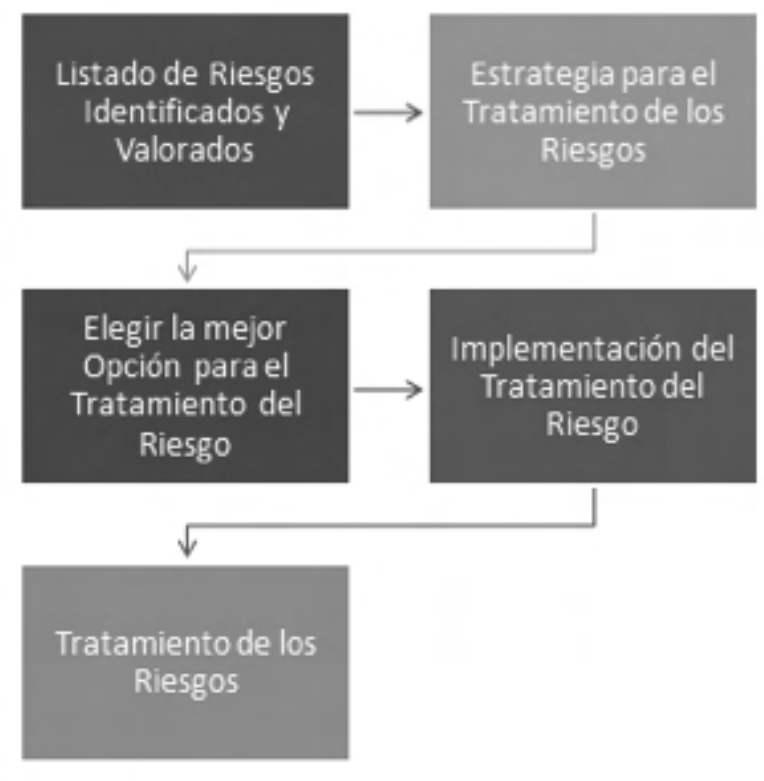

Figura 12.

Diagrama de flujo del proceso: "Tratamiento de los riesgos"

A continuación se ilustra, a través de un diagrama de flujo de datos, el proceso "Validación del modelo Mogripi" (Figura 13).

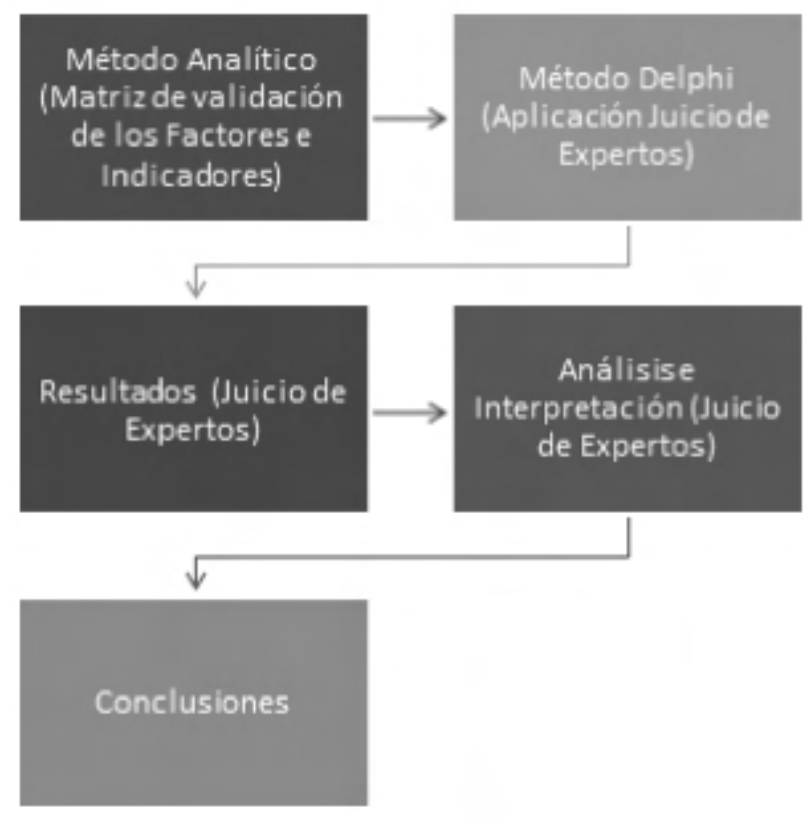

Figura 13.

Diagrama de flujo del proceso: "Validación del modelo Mogripi"

\section{Resultados}

Para la validación del modelo se realizaron dos rondas de aplicación del método Delphi. En la primera ronda los expertos se lograron obtener consenso de 7 indicadores de un total de 10, evidenciándose el alineamiento de los indicadores con las fortalezas del modelo Mogripi. Los indicadores 1, 3 y 6 registraron una desviación estándar por encima del valor fijado antes de iniciar la ronda (>4.5), por lo tanto se ejecuta una segunda ronda y más adelante se documentan los resultados de esta.

\section{Análisis de los indicadores planteados en el modelo Mogripi}

Indicador n. ${ }^{\circ}$ 1. ¿Se identifican los aspectos más relevantes fuera del proyecto que puedan influenciar los objetivos de este?

El modelo Mogripi hace clara referencia a la identificación de los diferentes aspectos que puedan influenciar los objetivos del proyecto, orientado a los más relevantes o críticos en un contexto externo.

Indicador n. ${ }^{\circ}$ 2. ¿Se identifican los aspectos más relevantes dentro del proyecto que puedan influenciar los objetivos de este?

El modelo Mogripi hace clara referencia a la identificación de los diferentes aspectos que puedan influenciar los objetivos del proyecto, orientado a los más relevantes o críticos en un contexto interno.

Indicador $n .^{\circ}$ 3. ¿Se define una estrategia para evaluar la importancia del riesgo?

Se define la estrategia a utilizar para evaluar la importancia del riesgo. Algunos de los aspectos a tener en cuenta son: naturaleza, causas, consecuencias, probabilidad, nivel de riesgo, apetito de riesgo y tolerancia al riesgo.

Indicador n. ${ }^{\circ}$ 4. ¿El modelo asocia los planes de GR del proyecto actual y los anteriores?

Dentro de la estrategia de la gestión del riesgo el modelo asocia dos entradas: los planes de GR del proyecto actual y los anteriores.

Indicador $\mathrm{n} .^{\circ} 5$. ¿Se identifican todos los riesgos posibles que puedan impactar positiva o negativamente el logro de los objetivos del proyecto?

El modelo contempla identificar la mayoría de riesgos posibles, elaborando una lista que permita determinar 
todos los aspectos que puedan impactar positiva o negativamente en el logro de los objetivos del proyecto.

Indicador n. ${ }^{\circ}$ 6. ¿En la identificación de los riesgos el modelo sugiere la categorización de estos (estructura de desglose de riesgos)?

Se deben identificar la mayoría de riesgos posibles, elaborando una lista que permita determinar todos los aspectos que puedan impactar positiva o negativamente en el logro de los objetivos del proyecto. Los riesgos se deben clasificar en categorías (estructura de desglose de riesgos). Los aspectos básicos a tener en cuenta son: origen de los riesgos, impacto, causas y consecuencias.

Indicador n. ${ }^{\circ}$ 7. ¿En el análisis de los riesgos se hace cualitativamente o cuantitativamente teniendo en cuenta el contexto del riesgo del proyecto?

El análisis puede ser cualitativo o cuantitativo dependiendo del contexto del riesgo del proyecto. Se deben tener en cuenta los aspectos que afectan a las consecuencias y la probabilidad.

Indicador n. ${ }^{\circ}$ 8. ¿La evaluación de los riesgos está orientada a establecer prioridades al momento del tratamiento del riesgo?

Una vez realizado el análisis de los riesgos estos deben ser valorados para determinar la prioridad al momento del tratamiento del riesgo.

Indicador n. ${ }^{\circ}$ 9. ¿El modelo establece criterios que permitan elegir la mejor opción o la más adecuada para el tratamiento del riesgo?

Se debe elegir la mejor opción o la más adecuada para el tratamiento del riesgo, se debe establecer una relación costo/beneficio de la opción a implementar.

Indicador n. ${ }^{\circ}$ 10. ¿El modelo sugiere la generación de un plan de tratamiento de riesgo documentado?

Se debe generar un documento con las acciones a realizar en el tratamiento de los riesgos. Los aspectos a tener en cuenta son: responsables, recursos, acciones propuestas y tiempo.

\section{Comentarios}

Al finalizar la presente investigación se obtuvieron resultados favorables o positivos alcanzados. Con base en estos resultados se propusieron algunas conclusiones. De igual forma, se dejan bases para el inicio de futuras investigaciones asociadas al objetivo principal de la investigación.

\section{Referencias}

Cocho, J. M. \& Adam, M. R. (2003). Estudio exploratorio sobre los métodos de gestión de proyectos de alto riesgo. Primer Congreso Soporte del Conocimiento con la Tecnología, SOCOTE, Valencia, España.

Chamoun, Y. (2002). Administración profesional de proyectos. La Guía. México D. F., México: McGraw-Hill.

Graham, R. \& Englund, R. (1999). Administración de proyectos exitosos. México D.F., México: Pearson, Addison Wesley.

Instituto Colombiano de Normas Técnicas y Certificación. [ICONTEC] (2011). Norma Técnica Colombia para la Gestión del Riesgo ISO 31000. Colombia.

Marcelo, J. \& Rodenes, M. (2003). Estudio exploratorio sobre los métodos de gestión de proyectos de alto riesgo. Primer Congreso Soporte del Conocimiento con la Tecnología, SOCOTE, Valencia, España.

Shari, P. (2008). Ingeniería de software. Buenos Aires, Argentina: Prentice Hall.

Software Engineering Institute. (2009). Risk Management. Pittsburgh, EE.UU: Carnegie Mellon University. Recuperado de http://www.sei.cmu.edu/risk/ Sommerville, I. (2006). Ingeniería de software. Madrid, España: Pearson, Addison Wesley. 